\title{
Low-Rank and Nonlinear Model Approach to Image Inpainting
}

\author{
Ryohei Sasaki*, Katsumi Konishi ${ }^{\dagger}$, Tomohiro Takahashi* and Toshihiro Furukawa* \\ * Graduate School of Engineering, Tokyo University of Science, Tokyo, Japan \\ $\dagger$ Department of Computer Science, Kogakuin University, Tokyo, Japan \\ Email: 4415612@ed.tus.ac.jp
}

\begin{abstract}
This paper proposes a new algorithm for image inpainting algorithm based on the matrix rank minimization with nonlinear mapping function. Assuming that each intensity value of a nonlinear mapped image can be modeled by the autoregressive (AR) model, the image inpainting problem is formulated as a kind of the matrix rank minimization problem, and this paper modifies the iterative partial matrix shrinkage (IPMS) algorithm and provides an inpainting algorithm, which estimates a nonlinear mapping function and the missing pixels simultaneously. Numerical examples show that the proposed algorithm recovers missing pixels efficiently.

Index Terms - image inpainting, matrix rank minimization, $\mathrm{AR}$ modeling, matrix recovery, manifold learning
\end{abstract}

\section{INTRODUCTION}

This paper deals with digital image inpainting, which is the technique of reconstructing small damaged portions of an image. Various methods have been proposed to achieve the recovery of missing pixels, and there two kinds of method. One is based on the image properties such as smoothness of images [1], [2], and the other algorithms recover images by interpolating the missing pixels using the intensity values of neighbor pixels [3], [4]. In [5], assuming that a textured image is modeled as the autoregressive (AR) model, the inpainting is achieved by identifying the AR model. In [6], [7], a textured image is modeled as a state space representation, matrix rank minimization approaches are proposed. The AR model and rank minimization based algorithm has been proposed in [8], which assumes that the value of each pixel is a linear combination of the neighbor pixels and estimates the missing values by minimizing a Hankel-like matrix. While the inpainting performance of AR model based algorithm depends on given order of AR model, this algorithm estimates missing intensity values and the model order simultaneously and therefore recovers missing pixels well.

This paper focuses on the AR model and rank minimization based algorithm [8] and provides a new low-rank approach to image inpainting. While the AR model based algorithms achieve high inpainting performance when the AR model is suitable for an image, actual images are not always modeled by the AR model properly, and an inpainting performance becomes worse. In order to improve a performance, this paper assumes that an image can be modeled by a nonlinear AR model, where nonlinear mapped image is modeled by a linear AR model, and provides a new algorithm based on a low-rank approach by modifying the iterative partial matrix shrinkage
(IPMS) algorithm [9]. The proposed algorithm estimates intensity values of missing pixels and a nonlinear mapping function simultaneously. Numerical examples show the effectiveness of the proposed algorithm compared with other algorithms.

\section{LOW-RANK AND LINEAR AR MODEL APPROACH}

This section introduces a low-rank and linear autoregressive (AR) model approach to image inpaiting based on the algorithm proposed in [8].

Let $x_{i, j}$ denote the intensity value of a 2D gray level image at $(i, j)$, where $1 \leq i \leq M$ and $1 \leq j \leq N$. We assume here that an image is modeled by the AR model where $x_{i, j}$ satisfies

$$
\sum_{l=-K}^{K} \sum_{m=-K}^{K} a_{l, m} x_{i+l, j+m}+d_{i, j}=0
$$

where $a_{0,0}=1$, and $d_{i, j}$ denote the modeling error, which is independent of $x_{i, j}$ and zero mean. This model can describe a texture of the image well and is used in several image inpainting algorithms such as [5]. Then the inpainting problem is to estimate the intensity values $x_{i, j}$ of the missing region using those of the known region. Let $\Omega$ and $\Omega^{c}$ denote the index sets of pixels in the known region and the missing region, respectively. If the model order $K$ is known, the image inpainting problem based on the AR model is formulated as follows,

$$
\begin{aligned}
\text { Find } & x_{i, j} \text { for }(i, j) \in \Omega^{c} \\
\text { s.t. } & x_{i, j}=x_{i, j}^{*} \text { for }(i, j) \in \Omega \\
& \sum_{i} \sum_{j}\left(\sum_{l=-K}^{K} \sum_{m=-K}^{K} a_{l, m} x_{i+l, j+m}\right)^{2} \leq \epsilon
\end{aligned}
$$

where $x_{i, j}^{*}$ denotes the intensity value of known region, and $\epsilon$ is a given constant which denotes the upper limit of the modeling square error. In this problem, design variables are $a_{l, m}$ and $x_{i, j}$ for $(i, j) \in \Omega^{c}$.

The problem (2) can be solved by estimating AR coefficients $a_{l, m}$ and missing variables $x_{i, j} \in \Omega^{c}$ alternately. However, the AR model order $K$ is usually unknown, and [8] reported that the algorithm does not work well when the model order $K$ is misestimated. Therefore, assuming the model order $K$ is small, [8] proposed an inpaiting algorithm estimating the 
missing values with low-order AR model. Let us define $\boldsymbol{x}_{i, j}$ and $X$ respectively by

$$
\begin{aligned}
& \boldsymbol{x}_{i, j}=\left[\begin{array}{cccc}
x_{i-K, j-K} & x_{i-K, j-K+1} & \cdots & x_{i-K, j+K} \\
x_{i-K+1, j-K} & x_{i-K+1, j-K+1} & \cdots & x_{i-K+1, j+K}
\end{array}\right. \\
& \text {... } \\
& \left.\begin{array}{llll}
x_{i+K, j-K} & x_{i+K, j-K+1} & \cdots & x_{i+K, j+K}
\end{array}\right]^{T} \\
& \in \mathbb{R}^{(2 K+1)^{2}},
\end{aligned}
$$

and

$$
\begin{array}{r}
X=\left[\begin{array}{ccc}
\boldsymbol{x}_{K+1, K+1} & \cdots & \boldsymbol{x}_{K+1, N-K} \\
\boldsymbol{x}_{K+2, K+1} & \cdots & \boldsymbol{x}_{K+2, N-K} \\
\cdots & & \\
\boldsymbol{x}_{M-K, K+1} & \cdots & \boldsymbol{x}_{M-K, N-K}
\end{array}\right]^{T} \\
\quad \in \mathbb{R}^{(M-2 K)(N-2 K) \times(2 K+1)^{2}} .
\end{array}
$$

The vector $\boldsymbol{x}_{i, j}$ consists of neighbor pixels of $x_{i, j}$ used in the AR model, and $X$ is the Hankel-like structured matrix whose row is $\boldsymbol{x}_{i, j}$. To simplify the notation, we define $\mathcal{X} \subset \mathbb{R}^{(M-2 K)(N-2 K) \times(2 K+1)^{2}}$ as the set of matrices of the structured matrix defined in (4). If the $d_{i, j}=0$ in (1), $X$ satisfies

$$
\operatorname{Rank}(X)=(2 K+1)^{2}-1 .
$$

Since $K$ is unknown and assumed to be small, the image inpainting problem is formulated by modifying (2) as follows,

$$
\begin{aligned}
\text { Minimize } & \operatorname{Rank}(Z) \\
\text { s.t. } & \|Z-X\|_{F}^{2} \leq \epsilon \\
& x_{i, j}=x_{i, j}^{*} \text { for }(i, j) \in \Omega \\
& X \in \mathcal{X}
\end{aligned}
$$

where $x_{i, j}$ is an element of $X$, which is defined according to (3) and (4). In this problem, $X$ and $Z$ are design variables.

Since the matrix rank minimization problem is NP hard in general, the problem (6) is defficult to solve. Then we usually apply the nuclear norm and Lagrange relaxations and obtain the following convex problem,

$$
\begin{aligned}
\text { Minimize } & \gamma\|Z\|_{*}+\frac{1}{2}\|Z-X\|_{F}^{2} \\
\text { s.t. } & x_{i, j}=x_{i, j}^{*} \text { for }(i, j) \in \Omega, \\
& X \in \mathcal{X}
\end{aligned}
$$

where $\gamma$ is a given constant, and $\|Z\|_{*}$ denotes the nuclear norm of the matrix $Z$, which is equal to the sum of its singular values. While the nuclear norm minimization is used in various low-rank approaches in signal processing, it causes signal distortion since the power of signals, which is equal to the sum of squared singular values, is reduced by minimizing its nuclear norm. To avoid this signal distortion, [9] proposes a problem of minimizing the sum of non-dominant singular values as follows,

$$
\begin{aligned}
\text { Minimize } & \gamma\|Z\|_{*, r}+\frac{1}{2}\|Z-X\|_{F}^{2} \\
\text { s.t } & x_{i, j}=x_{i, j}^{*} \text { for }(i, j) \in \Omega, \\
& X \in \mathcal{X}
\end{aligned}
$$

where $\|Z\|_{*, r}$ is defined as $\|Z\|_{*}=\sum_{i=r+1}^{(2 K+1)^{2}} \sigma_{i}(Z)$, and $\sigma_{i}(\cdot)$ denotes the $i$ th greatest singular value of a matrix. This problem is equal to the nuclear norm minimization when $r=0$ and can be solved by the iterative partial matrix shrinkage algorithm (IPMS) proposed in [9], which iterates the following update schemes,

$$
\begin{array}{ll}
\text { Step 1 } & Z \leftarrow \mathcal{T}_{r, \lambda}(X), \\
\text { Step 2 } & X \leftarrow \mathcal{P}_{\mathcal{X}, \Omega}(Z),
\end{array}
$$

where $\mathcal{T}_{r, \lambda}(X)$ denotes the partial soft thresholding operator replacing the $i$ th singular values of $X$ with $\max \left(\sigma_{i}(X)-\lambda, 0\right)$ for $i \geq r+1$, and $\mathcal{P}_{\mathcal{X}, \Omega}: \mathbb{R}^{(M-2 K)(N-2 K) \times(2 K+1)^{2}} \rightarrow \mathcal{X}$ denotes the orthogonal projector defined as

$$
\mathcal{P}_{\mathcal{X}, \Omega}(Z)=\begin{aligned}
\operatorname{argmin} & \|X-Z\|_{F}^{2} \\
\text { s.t. } & X \in \mathcal{X}, x_{i, j}=x_{i, j}^{*}
\end{aligned} .
$$

Since (8) requires the value of $r$ regarding with a matrix rank, the IPMS estimates a matrix rank $r$ during iterations by using the scheme,

$$
r \leftarrow \underset{\hat{r}}{\operatorname{argmax}} \sigma_{\hat{r}}(X) \text { s.t. } \sigma_{\hat{r}}(X) \geq \alpha \sigma_{1}(X),
$$

where $\alpha<1$ is a given constant. The details of the IPMS algorithm are written in [9], and its performance is also shown in [10] comparing with other algorithms for the matrix rank minimization problem.

While the problem (8) with the update scheme (9) works well and achieves high accuracy inpainting when a linear AR model is suitable, which can be seen in Section IV, a linear AR model (1) is not always suitable for actual images. In order to improve the inpainting performance, this paper proposes a low-rank and nonlinear AR model approach.

\section{LOW-RANK AND NONLINEAR AR APPROACH}

This section assumes that an image can be modeled by a nonlinear AR model, where a nonlinear mapped image is described by a linear AR model, and provides a new inpainting algorithm based on a row-rank approach.

Let us assume that an image is modeled by the nonlinear AR model where the intensity value $x_{i, j}$ satisfies

$$
\sum_{l=-K}^{K} \sum_{m=-K}^{K} a_{l, m} f\left(x_{i+l, j+m}\right)+d_{i, j}=0,
$$

where $f: \mathbb{R} \rightarrow \mathbb{R}$ is a nonlinear function. If we know $f$, we can apply the inpainting algorithm proposed in the previous section after transforming each $x_{i, j}$ by $f$. This paper deals with the case that $f$ is unknown. We make an additional assumption that the function $f$ and its inverse function $g:=f^{-1}$ can be approximated by polynomial as follows,

$$
f(x) \approx \sum_{i=1}^{p} v_{i} x^{i}
$$

and

$$
g(y)=f^{-1}(y) \approx \sum_{i=1}^{p} w_{i} y^{i} .
$$


Then an inpainting problrm for nonlinear AR model corresponding to (8) can be described as

$$
\begin{aligned}
\text { Minimize } & \gamma\|Z\|_{*, r}+\frac{1}{2}\|Z-Y\|_{F}^{2} \\
\text { s.t } & Y=\sum_{i=1}^{p} v_{i} X^{(i)}, X=\sum_{i=1} w_{i} Y^{(i)}, \\
& x_{i, j}=x_{i, j}^{*} \text { for }(i, j) \in \Omega \\
& X \in \mathcal{X}
\end{aligned}
$$

where $X^{(i)}$ and $Y^{(i)}$ denote the $i$ th element-wise power of $X$ and $Y$, respectively. In the above problem, $X, Y, Z$, $\boldsymbol{v}=\left[\begin{array}{llll}v_{1} & v_{2} & \ldots & v_{p}\end{array}\right]^{T}$ and $\boldsymbol{w}=\left[\begin{array}{llll}w_{1} & w_{2} & \ldots & w_{p}\end{array}\right]^{T}$ are design variables. Because $f$ and $g$ are not always described exactly by the $p$ th order polynomials, this paper provides the following Lagrange relaxation,

$$
\begin{aligned}
\text { Minimize } & E(X, Y, Z, \boldsymbol{v}, \boldsymbol{w}) \\
\text { s.t } & x_{i, j}=x_{i, j}^{*} \text { for }(i, j) \in \Omega \\
& X \in \mathcal{X}
\end{aligned}
$$

where $E$ is defined by

$$
\begin{aligned}
E(X, Y, Z, \boldsymbol{v}, \boldsymbol{w})= & \gamma\|Z\|_{*, r}+\frac{1}{2}\|Z-Y\|_{F}^{2} \\
& +\lambda_{y}\left\|Y-\sum_{i=1}^{p} v_{i} X^{(i)}\right\|_{F}^{2} \\
& +\lambda_{x}\left\|X-\sum_{i=1}^{p} w_{i} Y^{(i)}\right\|_{F}^{2},
\end{aligned}
$$

and $\lambda_{x}$ and $\lambda_{y}$ are given constants. In order to solve the problem (12), we modify the update schemes for IPMS algorithm by adding steps to estimate $\boldsymbol{v}$ and $\boldsymbol{w}$ by the least squares method and to update $X^{(i)}$ and $Y^{(i)}$ using their gradient of the objective function $E$. Finally this paper proposes an image inpainting algorithm as shown in Algorithm 1, where pow $(\cdot, i)$ denots the $i$ th element-wise power of a matrix, and $\operatorname{svd}(Y)$ denotes the operation of singular value decomposition. Algorithm 1 with $p=1$ is equal to the low-rank and linear AR model approach.

\section{NumERICAL EXAMPLES}

This section presents numerical examples to show the efficiency of the proposed algorithm. We used six test images selected from image database (http://testimages.tecnick.com) using MATLAB on a computer with $3.60 \mathrm{GHz}$ Core i7 and 16 GB RAM. We use the parameters in Algorithm 1 as $\hat{K}=3$, $\lambda_{y}=\lambda_{x}=1.0, \gamma=0.1, \mu=0.5, \varepsilon=1.0 \times 10^{7}, \alpha=1.0$, $\alpha_{\min }=1.0 \times 10^{3}, \eta_{\alpha}=1.005$ and $t_{\max }=2000$, which achieve the best performance. We examine the exemplar based image inpainting method [1] with the number of entries of each patch is $3 \times 3$, the null space alternatingly optimization (NSAO) based inpainting algorithm [8] with $\hat{K}=3$ and Algorithm 1 with $p=1,3,5$ and 7 .

Figures $1-6$ show the results of texture-like images and Fig 7 shows the results within the proposed method by $p=$ 1,3,5 and 7. In Fig 2, the exemplar method generates some noise like cracking in edge region, the NSAO based method misestimates with false-colors, and the proposed method with $p=7$ recovers the image better than others. Table I shows

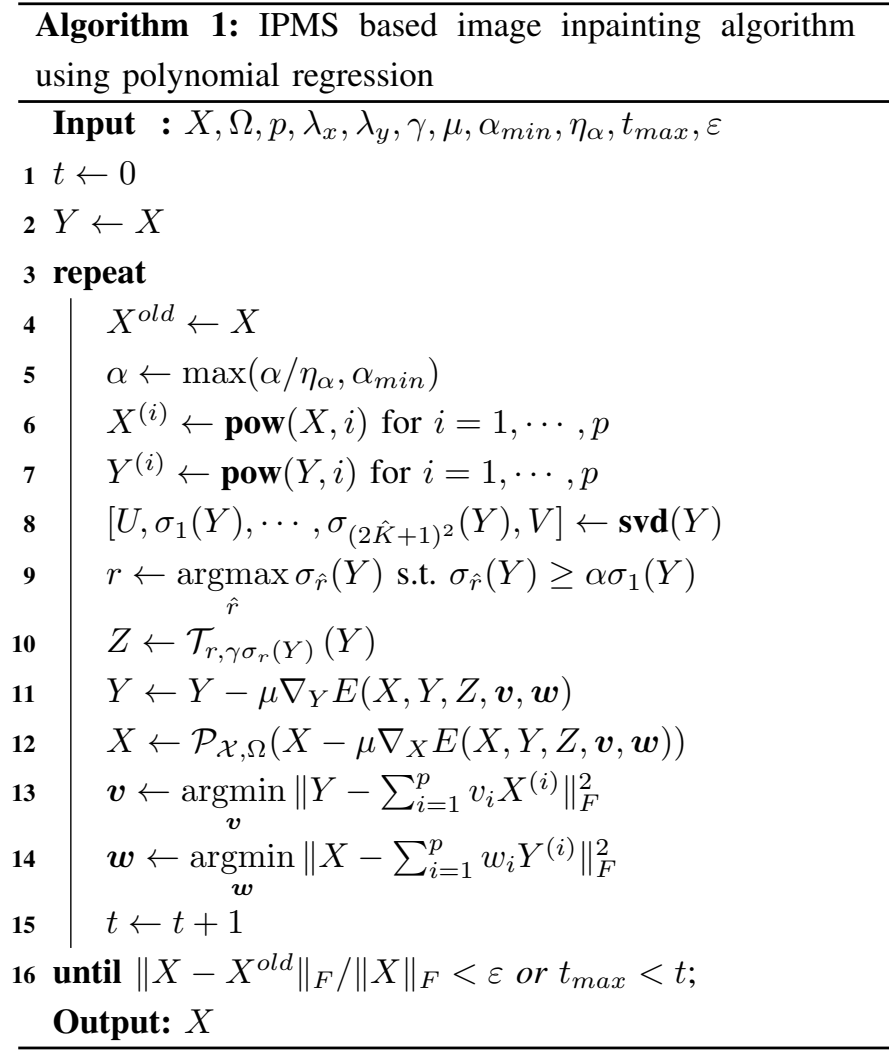

TABLE I

PERFORMANCE OF ALGORITHMS EVALUATED BY PSNR

\begin{tabular}{lccc}
\hline PSNR $[\mathrm{dB}]$ & ART 13 & ART 29 & ART 83 \\
\hline exemplar [1] & 36.30 & 27.64 & 29.97 \\
NSAO [8] & 41.41 & 25.83 & 30.94 \\
proposed $(p=1)$ & 41.38 & 26.84 & $\mathbf{3 1 . 7 9}$ \\
proposed $(p=3)$ & 43.23 & 26.96 & 31.79 \\
proposed $(p=5)$ & 43.81 & 27.35 & 31.79 \\
proposed $(p=7)$ & $\mathbf{4 3 . 8 9}$ & $\mathbf{2 7 . 8 1}$ & 31.78 \\
\hline & RGB 08 & RGB 39 & RGB 97 \\
\hline exemplar $[1]$ & 27.81 & 25.72 & 24.43 \\
NSAO [8] & 33.12 & 27.96 & 29.08 \\
proposed $(p=1)$ & 34.45 & 30.04 & 29.82 \\
proposed $(p=3)$ & 34.45 & 30.04 & 29.82 \\
proposed $(p=5)$ & 34.45 & 30.04 & 29.82 \\
proposed $(p=7)$ & $\mathbf{3 4 . 4 6}$ & $\mathbf{3 0 . 0 4}$ & $\mathbf{2 9 . 8 3}$ \\
\hline
\end{tabular}

performance of the algorithms evaluated by PSNR. We can see that the proposed algorithm achieves the best performance of all algorithms.

\section{CONCLUSion}

This paper proposes a new image inpainting algorithm which is based on the nonlinear AR model and estimates 


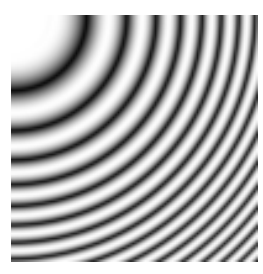

(a)

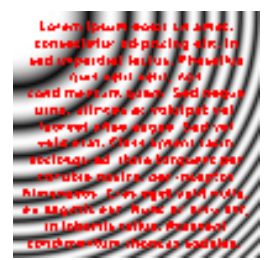

(b)

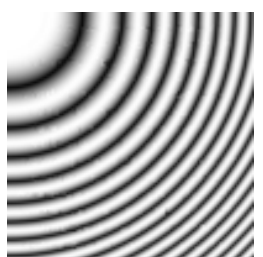

(c)
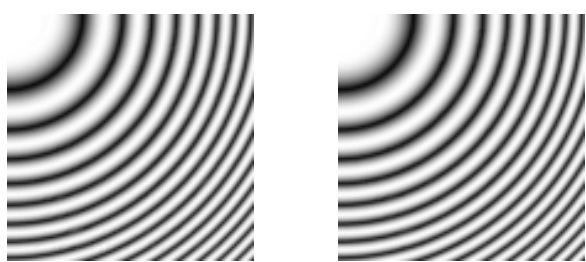

(d)

(e)

Fig. 1. Grayscale image ART 13: (a) original image, (b) inpainting area, inpainting results of (c) exemplar, (d) NSAO based algorithm and (e) the proposed algorithm with $p=7$.

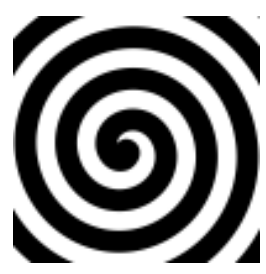

(a)

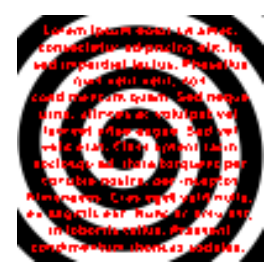

(b)

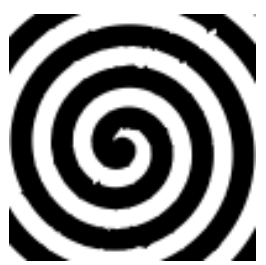

(c)

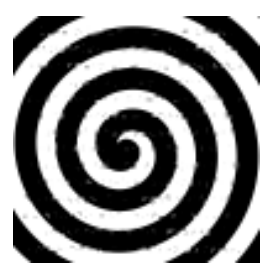

(d)

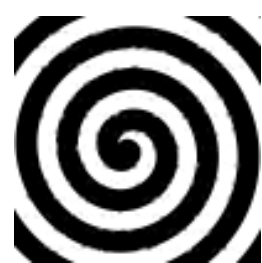

(e)

Fig. 2. Grayscale image ART 29: (a) original image, (b) inpainting area, inpainting results of (c) exemplar, (d) NSAO based algorithm and (e) the proposed algorithm with $p=7$.

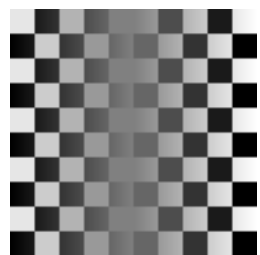

(a)

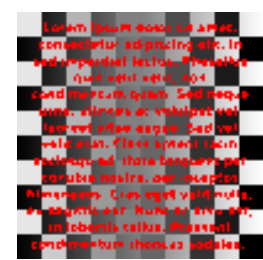

(b)

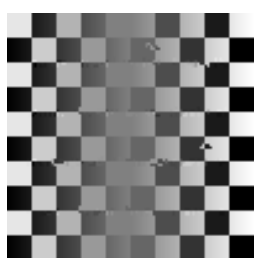

(c)

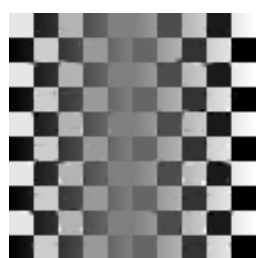

(d)

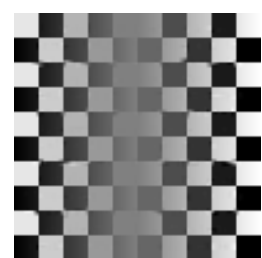

(e)

Fig. 3. Grayscale image ART 83: (a) original image, (b) inpainting area, inpainting results of (c) exemplar, (d) NSAO based algorithm and (e) the proposed algorithm with $p=7$.

the missing pixels with nonlinear function and the AR order simultaneously with matrix rank minimization.

In order to model the mapping function as nonlinear, we assumed that the function is approximated by a polynomial of a finite degree and the dimension of latent space which the mapped image patch belong to can be smaller than the space of the observed patch. The proposed inpainting algorithm is formulated as weighted nuclear norm minimization problem to approximate the rank minimization problem with the fitting error term. Numerical examples show that the effectiveness of the proposed algorithm.

\section{REFERENCES}

[1] A. Criminisi, P. Perez, and K. Toyama, "Region filling and object removal by exemplar-based image inpainting," IEEE Trans. Image Process., vol. 3, no. 9, pp. 1200-1212, 2004.

[2] B. Li, Y. Qi, and X. Shen, "An image inpainting method," Proc. IEEE Int. Conf. on Computer Aided Design and Computer Graphics, pp. 531-536, 2005.
[3] M. Bertalmio, G. Spario, V. Caselles, and C. Ballester, "Image inpainting," Proc. ACM SIGRAPH2000, pp. 417-424, 2000.

[4] A. Levin, A. Zomet, and Y. Weiss, "Learning how to inpaint from global image statics," Proc. IEEE Int. Conf. on Computer Vision, vol. 1, pp. 305-312, 2003.

[5] A. Kokaram, "A statistical framework for picture recon- struction using ar models," Image and Vision Computing, vol. 22, pp. 165-171, 2004.

[6] M. Sznaier and O. Camps, "A hankel operator approach to texture modeling and inpainting," Proc. of the 4th Int. Workshop on Texture Analysis and Synthesis, pp. 125-130, 2005.

[7] K. H. Jin, J. C. Ye, ”Annihilating filter-based low-rank Hankel matrix approach for image inpainting", IEEE Trans. Image Process., vol. 24, no. 11, pp. 3498-3511, 2015.

[8] T. Takahashi, K. Konishi, and T. Furukawa, "Rank minimization approach to image inpainting using null space based alternating optimization," IEEE International Conference on Image Processing, pp. 1717-1720, 2012.

[9] K. Konishi, K. Uruma, T. Takahashi and T. Furukawa, "Iterative partial matrix shrinkage algorithm for matrix rank minimization," Signal Processing, vol. 100, pp. 124-131, 2014.

[10] D. Lazzaro, "A nonconvex approach to low-rank matrix completion using convex optimization," Numerical Linear Algebra with Applications, vol. 23, no. 5, pp. 801-824, 2016. 


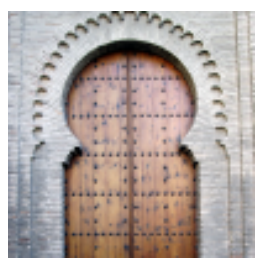

(a)

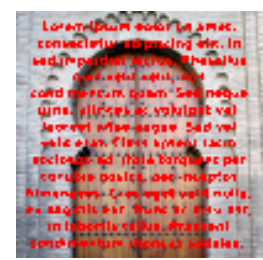

(b)

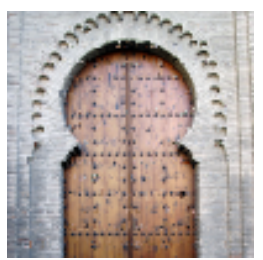

(c)

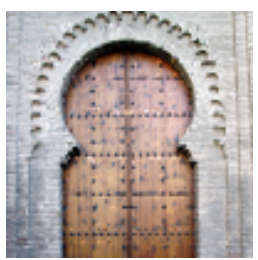

(d)

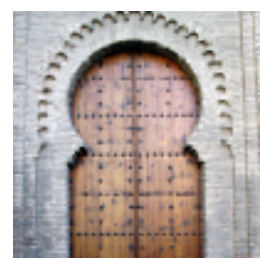

(e)

Fig. 4. RGB image RGB 08: (a) original image, (b) inpainting area, inpainting results of (c) exemplar, (d) NSAO based algorithm and (e) the proposed algorithm with $p=7$.

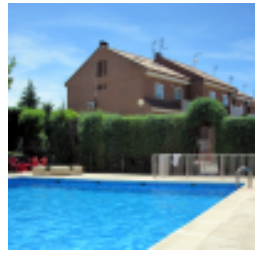

(a)

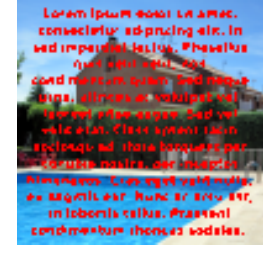

(b)

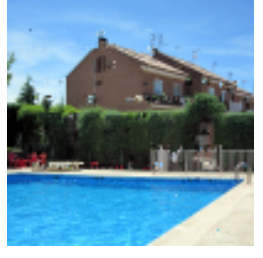

(c)

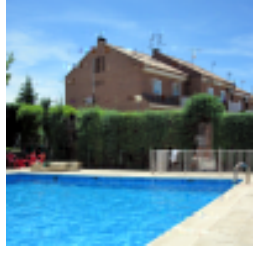

(d)

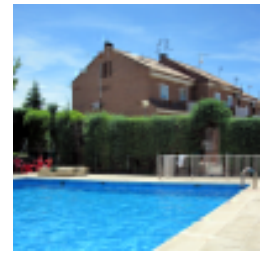

(e)

Fig. 5. RGB image RGB 39: (a) original image, (b) inpainting area, inpainting results of (c) exemplar, (d) NSAO based algorithm and (e) the proposed algorithm with $p=7$.

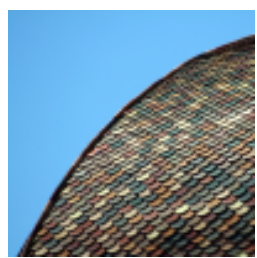

(a)

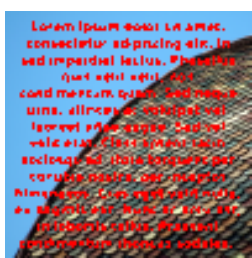

(b)

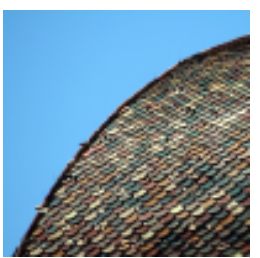

(c)

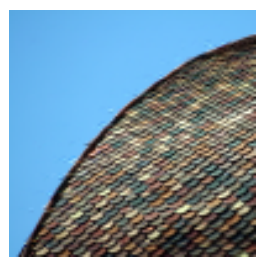

(d)

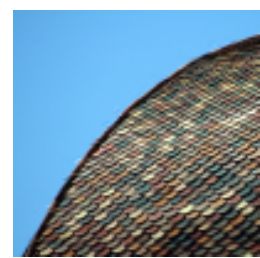

(e)

Fig. 6. RGB image RGB 97: (a) original image, (b) inpainting area, inpainting results of (c) exemplar, (d) NSAO based algorithm and (e) the proposed algorithm with $p=7$.

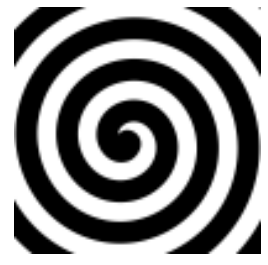

(a)

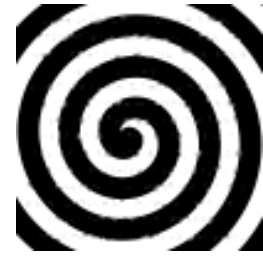

(b)

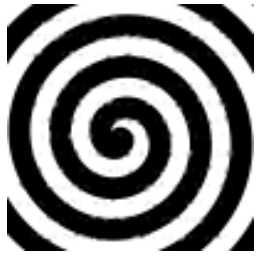

(c)

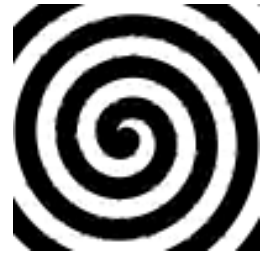

(d)

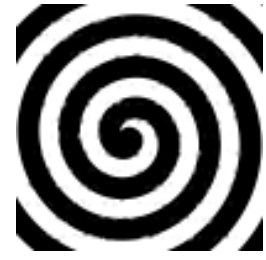

(e)

Fig. 7. Comparison of the parameter $p$ in Algorithm 1: (a) original image, (b) $p=1$, (c) $p=3$, (c) $p=5$ and (d) $p=7$. 